\title{
Principios metodológicos para trabajar la didáctica de las matemáticas en los grados de Educación Primaria de la UIC
}

\author{
M. Carme Balaguer FÀbregas \\ Universitat Internacional de Catalunya (España) \\ mcbalaguer@uic.es \\ Salvador VIDAL RAMÉNTOL \\ Universitat Internacional de Catalunya (España) \\ svidal@uic.es
}

\begin{abstract}
Resumen
Investigación en base a una programa matemático basado en la aplicación de seis principios psicopedagógicos escogidos a partir de un proceso cualitativo de análisis bibliográfico y curricular de las matemáticas de la Educación Primaria. El artículo justifica la aplicación de estos principios con el objetivo de dotar a los futuros maestros y maestras de instrumentos aplicables y de reflexión para que, en su tarea como docentes, ayuden a disminuir el fracaso escolar en matemáticas y aproximar estos contenidos a la vida cotidiana del alumnado.
\end{abstract}

Palabras clave: Educación Primaria; Matemáticas; Didáctica; Principios Metodológicos.

\section{Methodological principles for working with mathematics in Primary Education Degree (UIC)}

\begin{abstract}
Research based on a mathematical program based on six Psychopedagogical principles applying, chosen from a qualitative literature review process and mathematics curriculum of primary education. The paper justifies the application of these principles in order to equip future teachers whith applicable instrument for thought in their work as teachers, help reduce school failure in math and bring this content to everyday life of students.
\end{abstract}

Key Words: Primary Education; Mathematics; Teaching; Methodological Principles

\section{Referencia normalizada:}

Balaguer Fábregas, M. C.; Vidal Raméntol, S. (2013) Principios metodológicos para trabajar la didáctica de las matemáticas en los grados de Educación Primaria de la UIC. Historia y Comunicación Social. Vol. $18 \mathrm{~N}^{\circ}$ Especial Noviembre. Págs. 63-74.

Sumario: 1. Introducción. 2. Metodología. 3. Análisis y discusión. 4. Conclusiones. 5. Referencias bibliográficas. 6. Notas. 


\section{Introducción}

Según los resultados de los últimos informes PISA (2009), las competencias matemáticas constituyen uno de los factores que necesitan más refuerzo en los procesos de enseñanza y aprendizaje, para combatir el fracaso escolar. Por este motivo, existen muchas experiencias encaminadas a mejorar la didáctica de esta materia, que se transmiten en las facultades de Educación. La experiencia que argumentamos pretende avanzar en la búsqueda de un modelo susceptible de ser ajustado contingentemente a las características y necesidades del alumnado, de tal manera que se consiga que las matemáticas se conviertan en una materia significativa para ellos. Las teorías del aprendizaje social y del aprendizaje cognitivo ofrecen diferentes perspectivas de la enseñanza y el aprendizaje con muchas posibilidades de aplicación individualizada, gracias a la importancia que se ha demostrado que tienen los elementos manipulables del entorno, como son las interacciones entre los actores del hecho educativo y el diseño de las tareas y entornos educativos.

Es cierto también que las políticas educativas han ido recogiendo en forma de ley aplicable, todas las condiciones que se consideran adecuadas para evitar el fracaso escolar, por lo tanto, tenemos acceso a muchas informaciones sobre las condiciones educativas que necesita el alumnado, tantas, que en muchas ocasiones parece difícil tenerlas todas en cuenta en el momento de diseñar un programa de implementación curricular.

La selección teórica socio constructivista en la que se fundamenta el presente trabajo, Vigotsky (1979), Best (2001), Bruner (1997), Piaget (1985), Wells (2001), Rogoff (1993), etc. nos permite llegar a la conclusión de la complejidad implícita de las relaciones entre alumnado y profesorado. Complejidad que hay que extender a cada uno de los niños y niñas de cada grupo escolar. En base a esta selección se ha optado por elaborar una propuesta que contemple la aplicación de seis principios metodológicos pedagógicos que ayuden a sistematizar las interacciones en el aula y las implementaciones de los contenidos matemáticos.

\section{Metodología}

El objetivo general de esta investigación es analizar la bibliografía actual y el currículum para seleccionar los principios metodológicos que han de guiar el desarrollo de las didácticas de las matemáticas. Por lo tanto se trata de una investigación cualitativa de análisis bibliográfico. 


\section{Análisis y discusión}

Los principios escogidos se transfieren a la práctica a través de un proceso de enseñanza y aprendizaje estratégico (Monereo, 1999), que favorece la sistematización en su aplicación. Los principios seleccionados son los siguientes.

\subsection{Prevención}

La consideración tradicional que se tiene de las matemáticas como materia compleja implica que la presencia de niños y niñas con problemas para su comprensión se vea dentro de la normalidad. La aplicación de ayudas complementarias, permite a ese tipo de alumnado la superación con éxito de la competencia en matemáticas. Por este motivo, la inclusión de medidas instruccionales ordinarias, aplicadas al grupo entero favorece a aquellos casos que necesiten algún tipo de ayuda y que no estén prescritos. Asimismo también se propone presentar unas actividades más pautadas que permitirán mejorar el rendimiento del alumnado que, en principio, progresa adecuadamente en su desarrollo académico. Se han estudiado diferentes fórmulas para ayudar niños y niñas con dificultades de aprendizaje de las matemáticas, algunas de estas dificultades están descritas por Wright (1996). En primer lugar, la autora emplaza al profesorado a conocer a los niños perfectamente i destacarles sus puntos fuertes, para reforzar el conocimiento de su autoeficacia. Considera básico que no pierdan nunca la conexión entre la tarea y su significado y también que hagan oralmente sus estimaciones, antes de empezar las operaciones. Por otro lado, también destaca la importancia del trabajo en pequeños grupos, debido a las ventajas que tiene recibir ayudas adecuadas a su nivel de interacción simétrica.

\subsection{Atención a la diversidad}

La no segregación de los niños y niñas, posibilita, desde una perspectiva psicopedagógica, la administración de ayudas espontáneas entre compañeros, que es una medida muy válida a nivel de inclusividad. La metodología estratégica incluye material que incorpora guías de naturaleza metacognitiva que tienen por objetivo aportar estrategias de comprensión a aquellos alumnos que no las han adquirido espontáneamente. Gracias a esta medida, se consigue que un material se convierta en multinivel y pueda ser seguido por niños y niñas con distintos ritmos de aprendizaje. Permitiendo un margen de diferencia temporal y planificando actividades asociadas en forma de consultas o rincones de actividad para quienes van más rápido, nos aseguramos que cada niño o niña pueda finalizar su tarea dentro de la misma aula.

La interacción y el nivel de los diálogos entre alumnado y entre profesor/a y alumnos es un tema fundamental para atender a la diversidad. La adaptación al lenguaje de cada niño dentro de la normalidad del aula y la verificación de la comprensión son garantías de éxito en el aprendizaje. Por este motivo, se considera de suma importancia el trato verbal i el material curricular igualitario para todo el alumnado. Hay que intentar que las expectativas generadas sean positivas para todos los casos, porque la motivación hacia la correcta resolución de las tareas es el mejor motor para el aprendi- 
zaje. Otra acepción del concepto de atención a la diversidad es la consideración de la multiculturalidad, - sobre todo en relación a los contenidos de aprendizaje- y la idea que otras culturas tienen de la matemática. Hay autores como Alsina y Planas (2008), que sostienen que los conceptos matemáticos son comunes y que la única diferencia es el lenguaje, otros como Freire (1993) consideran que las necesidades propias de cada cultura priorizan diferentes conocimientos. En cualquier caso, la consideración multicultural de las matemáticas no se tiene que reducir a una traducción verbal de los contenidos, sino que tiene que abarcar un aspecto social y cultural más amplio que permita la comprensión de las diferentes necesidades que aquella persona tenía como elementos constitutivos de su forma de vida anterior.

\subsection{Motivación}

Vidal (2005: 27) define la motivación como

"una energía que orienta la conducta humana hacia un objetivo, y es susceptible de ser activada o desactivada. Si se activa produce motivación intrínseca o extrínseca, pero si es desactivada produce insatisfacción".

Hay muchas teorías de la motivación que han intentado dar respuesta a este fenómeno. Por su vinculación con la opción teórica de enseñanza y aprendizaje escolar adoptada en el presente artículo, nos centraremos en las orientaciones interactivas y cognitivistas de la motivación. Los maestros y maestras tienen la posibilidad de condicionar las demandas de las tareas de manera que sean percibidas como exitosas por el alumnado. La manera más eficaz de elaborar estas demandas es aplicar criterios de internalidad, estabilidad y control en los términos que se detallan a continuación:

\subsubsection{Atribución interna del éxito}

Aunque el origen de las tareas es externo, se han intentado buscar temas que los niños y niñas puedan percibir como muy próximos e interesantes. El trabajo sobre contenidos cercanos da significatividad al trabajo de los niños y niñas, con lo que se incrementan las posibilidades de éxito.

\subsubsection{Estabilidad del éxito en la tarea}

El alumnado asume que el éxito en la realización de una tarea es estable, cuando son conscientes de su utilidad. Es decir cuando pueden entender que aquello que aprenden podrá ser útil en su vida. La funcionalidad de los aprendizajes, se hace evidente cuando se proponen actividades en forma de problemas o situaciones problemáticas que de per sí, necesitan la presencia y la ayuda de los adultos. Cuando les damos ayudas e instrumentos que favorezcan su autonomía estamos introduciendo un elemento indiscutiblemente motivador porqué les permite entrar en una nueva dimensión cada vez más estable de su libertad personal. Enseñar como aprender da más independencia educativa que sencillamente enseñar qué aprender. 


\subsubsection{El lugar de control del éxito}

Cuando un alumno o alumna percibe que la actividad es accesible a sus competencias, tiende a desarrollar mecanismos de control autónomo e interno para su realización, y persiste hasta la resolución de la tarea. Cuando una actividad es percibida como inaccesible es muy fácil que se abandone. La motivación añadida que da la propia competencia en una tarea provoca la generación de expectativas positivas hacia ese trabajo. Todo ello permite introducirse en una espiral positiva de autoeficacia que alimenta la motivación intrínseca o motivación parar saber.

En relación al logro de la motivación intrínseca, Vidal (2005) cita diferentes estrategias y técnicas de motivación en el aula, que quedan determinadas en la tabla 1. Todas las modalidades tienen un doble objetivo: en primer lugar, conseguir un clima más agradable de trabajo que facilite la tarea y en segundo lugar conseguir motivar al alumnado para que, además de trabajar con tranquilidad, vayan cogiendo interés en lo que están aprendiendo, de manera que los aprendizajes sean significativos y permanentes, en lugar de memorísticos y efímeros.

Tabla 1. Estrategias y técnicas de motivación en el aula. (Adaptado de Vidal, 2005)

\begin{tabular}{|c|c|}
\hline \multirow{8}{*}{$\begin{array}{l}\text { Estrategias para } \\
\text { conquistar la atención }\end{array}$} & Comunicar la justificación de los objetivos \\
\hline & Despertar la curiosidad \\
\hline & Crear disonancias o "choques" \\
\hline & Modificar el medio físico de aprendizaje \\
\hline & Variar las condiciones de la instrucción \\
\hline & Variar los canales sensoriales \\
\hline & Usar el movimiento \\
\hline & $\begin{array}{l}\text { Usar sistemas de comunicación de forma } \\
\text { matizada }\end{array}$ \\
\hline \multirow{5}{*}{$\begin{array}{l}\text { Estrategias para } \\
\text { conquistar la } \\
\text { participación }\end{array}$} & Interrogatorio \\
\hline & Refuerzo positivo \\
\hline & Retroalimentación \\
\hline & Expectativas éxito crecientes \\
\hline & Representación de roles \\
\hline
\end{tabular}

Para captar o conquistar la atención, se hace siempre explícito el objetivo de aprendizaje en todas las actividades, lo que permite un efecto concomitante con las siguientes estrategias de despertar la curiosidad y la creación de disonancias. Por otro lado, el cambio de escenario de aprendizaje se puede realizar de diferentes maneras: impulsando la consulta externa de información diversa; buscando en internet en el aula de informática, etc. En cualquier caso, y según las circunstancias del entorno educativo se pueden buscar espacios que tengan interés y relación con los contenidos 
de aprendizaje. Respecto a la variación de las pautas de instrucción, podemos afirmar que por su naturaleza, la secuencia metodológica (Monereo, 1999), propia de la enseñanza estratégica ya comporta diferentes cambios instruccionales, relativos al trabajo individual, grupal, guiado o modelado, por lo tanto también es una condición contemplada en este programa. Igualmente se han intentado variar los canales sensoriales de entrada de la información, siempre considerando las limitaciones que pueda tener el contenido matemático. Se trata de presentar la información con diferentes formatos para que pueda ser entendida por niños y niñas con diferentes estilos de aprendizajes.

Las dos últimas estrategias se vinculan más a aspectos personales de estilo de instrucción del profesor o profesora que tiene que impartir la asignatura. Es preciso añadir que todos los programas de formación del profesorado ponen mucho énfasis en la adopción de modelos asertivos de interacción, de manera que la creación de climas positivos de enseñanza y aprendizaje sea un hecho.

Sobre las estrategias de conquista de la participación, normalmente se consiguen cuando se ha podido captar la atención inicialmente. En cualquier caso, una de las pautas sobre interacción oral más importante es fomentar constantemente el diálogo entre alumnos y entre profesora o profesor y entre el alumnado, de manera que el trabajo en el aula se fundamente en la resolución de conflictos cognitivos. Mantener una cierta tensión y alerta en el aula es un requisito para fomentar un estado de consciencia que favorezca la reflexión y la toma de decisiones sobre la tarea. Por este motivo, utilizar estrategias como la interrogación, la realimentación y el refuerzo positivo para alimentar las expectativas de éxito, son siempre elementos que propician el aprendizaje, y que es preciso utilizar, como hemos comentado anteriormente, como componentes del estilo instruccional del profesorado.

\subsection{Fomento del pensamiento crítico}

Alejarnos del aprendizaje automatizado y entrar en el aprendizaje consciente. Quizás esta sería la máxima deseable para llegar a formar sujetos responsables. Pero para llegar hasta aquí no es suficiente con enseñar a entender y a resolver un problema, es imprescindible que se enseñe a identificar los problemas. Cuando se consigue que los alumnos y alumnas sean capaces de identificar problemas, entenderlos y resolverlos, podremos afirmar que están construyendo un pensamiento crítico.

Según Morin (2000) el pensamiento crítico es aquel que estimula la formulación de preguntas pertinentes i la búsqueda de soluciones adecuadas. Los instrumentos a utilizar para llegar a este pensamiento crítico son simplemente: escuchar, hablar, leer y escribir. Estamos rodeados de situaciones problemáticas que son susceptibles de ser solucionadas matemáticamente. Si somos capaces de identificarlas como tal y de analizar sus elementos, también seremos capaces de resolverlos. Hay que redactar los enunciados de manera que fomenten el pensamiento crítico. Según Alsina y Planas (2008:18-19), la UNESCO publicó el año 1997 las directrices que tendría que seguir la educación crítica, en base al estudio de Sylvia Downs (1987). Este documento 
describe las competencias que tienen que alcanzar un alumno o alumna gracias a un aprendizaje basado en la resolución crítica de problemas y son los siguientes:

- El alumno se ha de responsabilizar de su aprendizaje y adoptar un papel activo.

- Ha de saber distinguir entre lo que hay que memorizar y lo que hay que entender.

- Ha de tomar decisiones y hacer preguntas para asegurarse la comprensión.

- Sentirse seguro para aprovechar las nuevas oportunidades de aprendizaje.

- Darse cuenta de las dificultades que se le presentan y de sus causas.

De entre muchas versiones sobre cuáles son las destrezas que hay que potenciar para desarrollar el pensamiento crítico, Bruning et al. (2005) optan por destacar los siguientes elementos: Conocimiento, inferencia, evaluación y metacognición.

No es posible aprender a pensar en abstracto, por lo tanto el pensamiento crítico se adquiere a partir del conocimiento concreto, i al mismo tiempo un nuevo conocimiento emerge como producto del pensamiento crítico.

La inferencia también es un elemento trabajado en el programa. Se fomenta la inferencia deductiva cada vez que planteamos actividades en las cuales el alumnado tiene que buscar una conclusión específica a partir de la información dada. Se fomenta también la inferencia inductiva cuando pedimos la extracción de conclusiones generales -en muchos casos hipotéticas- que se pueden desprender de un hecho concreto.

La evaluación, en el caso de las actividades matemáticas de primaria la enfocaremos desde dos puntos de vista. En primer lugar, se intenta promover que las actividades precisen del mantenimiento del control de la tarea por parte del alumnado, es decir, que se facilite la rectificación si se considera necesario. En el control de la tarea se consideren integradas subdestrezas como pueden ser el análisis de las respuestas y la valoración de su adecuación a la demanda, ambas vinculadas a la capacidad de evaluar. Y, en segundo lugar se fomenta también el ejercicio de autoevaluación personal. Con ello se quieren potenciar las disposiciones personales en relación a valores como la sinceridad, la honestidad y la iniciación en el juicio sobre la propia actuación.

En último lugar, la metacognición, como capacidad óptima para fomentar el pensamiento crítico, está presente en el programa desde el principio como elemento que favorece la consciencia en el aprendizaje.

La estructura de este modelo instruccional pretende tratar la resolución de problemas matemáticos a partir del planteamiento de situaciones y contenidos conocidos por los alumnos, pero interpretables, de manera que per un lado se estimula el diálogo y la discusión sobre el contenido de la demanda y por otro lado el profesor o profesora modela verbalmente como hace sus procesos de razonamiento para llegar a la solución. Atención al modelaje porqué tiene que tomarse como una alternativa de pensamiento adulto, en ningún caso como una solución única y prescriptiva. Para fomentar el pensamiento crítico, es imprescindible abrirse al diálogo. 
Alsina y Planas (2008) destacan algunas actividades y actuaciones para la mejor del pensamiento crítico desde la matemática, que desarrolló el grupo EMAC ${ }^{1}$ el año 2005. Con la premisa que no es posible aprender a pensar críticamente en abstracto, los autores pensaron en contenidos concretos que generasen conflicto entre el alumnado, con la finalidad de estimularles a hacer preguntas, suposiciones, dudas, avanzar hipótesis, etc. Son contenidos cercanos como los productos light, un proyecto urbanístico que afecta un pueblo, los planos de un apartamento, etc., en cualquier caso, como citan sus autores:

\begin{abstract}
"Una actividad no es crítica sólo por su enunciado, sino que lo es por la dinámica que se crea alrededor de su resolución. Esta dinámica tiene que sugerir cuestiones, curiosidades y dudas, sin que ello signifique necesariamente llegar a conclusiones. Cuando el pensamiento crítico no lleva a resolver problemas o a extraer conclusiones, también es productivo." (Alsina i Planas, 2008:48)
\end{abstract}

\title{
3.5 Contextualización y globalización
}

Cuando estudiamos cualquier conocimiento matemático, lingüístico, social, etc., no nos podemos restringir a su epistemología, sino que hay que trabajar también su relación con el mundo y su relación con las demás materias. Hablamos de contextualización cuando nos referimos a la relación de un conocimiento con el mundo y hablamos de globalización cuando nos referimos a su relación con las demás áreas curriculares. Ambos conceptos nos remiten a una situación importante en la enseñanza y el aprendizaje, que es la significatividad y el sentido de los aprendizajes. Edgar Morin (2000) afirma que la contextualización es imprescindible para no caer en la trampa de considerar el conocimiento (matemático o de cualquier naturaleza) como un conjunto de informaciones ajenas a la realidad. También Alsina y Planas (2008) dicen que el conocimiento matemático es un claro ejemplo de necesidad de ser contextualizado, ya que la simbología propia del lenguaje matemático, adquiere un sentido específico en el ámbito matemático, muy diferente del que los mismos símbolos puedan tener en otros ámbitos. En relación a la globalización curricular, partimos de una premisa favorable a este concepto: se intenta demostrar la importancia de leer bien para mejorar el rendimiento matemático. Alsina i Planas (2008) asumen que el desarrollo conjunto de ciertas habilidades mejora la comprensión de estas habilidades en comparación a cuando se tratan separadamente. Un ejemplo aplicado a niños y niñas de primaria: si se trabaja la estadística o la organización de la información junto a conceptos de demografía próxima, es muy posible que estos niños acaben comprendiendo a la vez, aspectos propios de las dos materias que impartidos separadamente no habrían sido capaces de comprender y vincular.

\subsection{Enseñanza y aprendizaje estratégico}

La aplicación de la secuencia metodológica (Monereo, 1999) permite llevar a la práctica los principios educativos que estamos detallando. Cada uno de los pasos de la citada secuencia conlleva una carga pedagógica acumulativa que podemos asociar al complimiento de estos principios. El primer paso de la secuencia metodológica es 
el modelaje cognitivo. Este paso consiste en la ejemplificación que hace un experto sobre "como piensa" mientras realiza la tarea. Con el modelaje cognitivo, el profesor o la profesora enseña como tomar consciencia del trabajo y como tomar decisiones sobre el camino a seguir para llegar a un buen resultado. El profesor o la profesora, como modelo de actuación, facilita la previsión y rebaja la angustia que puede provocar una nueva tarea. Todo ello permite incrementar las expectativas de éxito del alumnado. El segundo paso es la práctica guiada. Esta práctica la vinculamos con la prevención y la atención a la diversidad, ya que permite a las niñas y niños trabar según diferentes ritmos de aprendizaje. Esto es posible gracias a la existencia de una pauta que garantiza la correcta ejecución de los pasos del problema. El tercer paso es la práctica en contextos variados, lo que nos garantiza la inclusión de los principios de globalización y contextualización. Globalización porqué integramos diferentes materias al aprendizaje matemático como son la lectura, la geografía, el conocimiento del medio social, etc. Y contextualización porqué se sitúa la acción educativa en el entorno más cercano y conocido del alumnado. Tal y como recoge Riviere (2003), la práctica de las matemáticas no se puede limitar al ámbito cognitivo porqué, en primer lugar, dentro de sus objetivos se contempla el desarrollo de capacidades asociadas a la comunicación o a la comprensión del medio social, y en segundo lugar, porqué la enseñanza y el uso de las matemáticas se realiza y se deberá realizar en un medio social real. El cuarto paso es la práctica en grupos cooperativos. Esta tarea, en primer lugar, favorece la interacción entre iguales, hecho muy motivador porqué libera al alumnado de la formalidad más o menos intensa generada por las relaciones con el adulto. En segundo lugar es un espacio interesante de atención a la diversidad en relación a la combinación de distintos ritmos de aprendizaje. Y en tercer lugar, al tratarse de una técnica basada en el debate y en el consenso, se produce un buen ejercicio de desarrollo del pensamiento crítico. El último paso de la secuencia metodológica es la práctica independiente y representa la comprobación de la asimilación de aprendizaje y de la autonomía en la ejecución de las tareas. En esta práctica queda reflejado el trabajo y la reflexión personal. Supone un ejercicio doble, por un lado conocer la propia autoeficacia y por otro lado desarrollar el pensamiento crítico, elementos que acabaran de certificarse cuando se respondan los ítems de autoevaluación.

\section{Conclusiones}

La propuesta de principios metodológicos tienen que contemplar dos acepciones para que sea aplicable. En primer lugar, el docente o la docente debe tener siempre consciencia del significado de estos conceptos y voluntad para aplicarlos. Y en segundo lugar es preciso trabajar con un procedimiento que ayude a garantizar su aplicación gracias a su uso sistemático. Consecuentemente a estas premisas, afirmamos que la puesta en práctica de actividades estratégicas, a partir de pasos previamente diseñados, permite cumplir con los principios pedagógicos seleccionados y también dotar al alumnado de instrumentos que tienen por objetivo mejorar las habilidades cognitivas y metacognitivas. Trabajar con un enfoque didáctico que permita 
un mantenimiento sostenido de la consciencia del aprendizaje, contribuye al desarrollo de las competencias que nos demanda el currículum. Como afirma Badia (2003), la utilización de estrategias de aprendizaje de manera ordinaria, permite que estas sean asimiladas por los alumnos como un proceso cognitivo transversal, independientemente del contexto donde se vayan aplicando. De esta manera, este proceso es susceptible de ser utilizado y transferido a otro tipo de contenidos y situaciones de aprendizaje, hasta que se determina que los sujetos han alcanzado la autonomía en la tarea.

\section{Referencias bibliográficas}

Libros

ALSINA, Àngel y PLANAS, Núria. (2008): Matemática inclusiva. Propuestas para una educación matemática accesible. Madrid. Narcea.

BADIA, Antoni (coord.) (2003): Actividades estratégicas de enseñanza y aprendizaje. Propuestas para fomentar la autonomía en el aprendizaje. Barcelona. CEAC.

BEST, John B. (2001): Psicología cognitiva. Madrid. Paraninfo.

BRUNER, Jerome ( (1997): La educación, puerta de la cultura. Madrid. Visor.

BRUNING, Roger H., SCHRAW, Gregory J., NORBY, M.M. y RONNING, Royce R. (2005): Psicología cognitiva y de la instrucción. Madrid. Pearson.

DEWEY, John (1910): How we think. New York. Cosimo classics

FREIRE, Paolo (1993): Pedagogía de la esperanza. Buenos Aires. Siglo XXI editores.

MONEREO, Carles (coord.) (1999): Estrategias de enseñanza y aprendizaje. Formación del profesorado y aplicación en la escuela. Barcelona. GRAO.

MORIN, Edgar (2000): Els set coneixements necessaris per a l'educació del futur. Barcelona: centre UNESCO de Catalunya.

PIAGET, Jean (1985): El nacimiento de la inteligencia en el niño. Barcelona. Crítica. WELLS, Gordon (2001): Indagación dialógica. Barcelona. Paidós

RIVIERE, Ángel V. (2003). Las matemáticas en el desarrollo de las capacidades de los alumnos. En MARTÍN et al: Aprender contenidos, desarrollar capacidades. Intenciones educativas y planificación de la enseñanza. Barcelona. Edebé.

ROGOFF, Bárbara (1993): Aprendices del pensamiento. El desarrollo cognitivo en el contexto social. Barcelona. Paidós.

VIDAL, Salvador (2009): Estrategias para la enseñanza de las matemáticas en secundaria. Barcelona. Laertes.

VIDAL, Salvador (2013): El día del número, motivación de la matemática. Actuación de éxito. Saarbrücken. Publicia.

VIGOTSKY, Lev (1979): El desarrollo de los procesos psicológicos superiores. Barcelona. Crítica. 
Revistas en papel

VIDAL, S. (2010): La comunicación en la didáctica de las matemáticas. Vivat Academia. UCM 112, p. 1-9

VIDAL, S. (2010): Talens dag att skapa lust för matematiklärande. Nämnaren Tidskrift för matematikundervisning (Göteborgs Universitet), 173 (1), p. 43-46.

VIDAL, S. (2011): Good morning, numbers day. Australian Primary Mathematics Classroom. (The Australian Association of Mathematics Teachers Inc). 16 (3), p. 25-28.

VIDAL, S (2011): La situació de la didàctica de les matemàtiques a la secundària catalana. Anàlisi de l'estat de l'ensenyament i l'aprenentatge. Temps d'Educació, (Universitat de Barcelona), 41, p. 185-199.

VIDAL, S. BALAGUER, C. (2013): La comunicación de los problemas de matemáticas en la didáctica de los Grados de Educación en la UIC. Estudios sobre el Mensaje Periodístico, UCM, 19,p. 531-541

VIDAL, S. FUERTES, M.T (2013): La dinámica de grupos para el trabajo cooperativo facilita la comunicación. Vivat Academia, UCM, 123, p.1-12

Artículos en publicaciones web

CALDEVILLA DOMÍNGUEZ, David (2009): Comunicar en situaciones de crisis, en Revista de Comunicación Vivat Academia, n 105. Disponible en: www.ucm. es/info/vivataca/numeros/n105/innovaci.htm\#david. [12-3-2011]

GENERALITAT DE CATALUNYA : Decret d'Ordenació de l'Ensenyament Primari del Departament d'Educació de la Generalitat de Catalunya (Decret 142/2007 de 26 de juny, DOGC núm. 4915 - 29/06/2007) [10-6-11]

OCDE (2010): Pisa 2009 results. Paris: OCDE, vols. I, II, III, IV y V. http://www. oecd.org/document/51/0,3746,en_32252351_32235731_39732595_1_1_1_1,00. html [15-06-11]

URRUTIKOETXEA OLATABERRI, Gorka (2010): Crisis en el modelo educativo actual, en Revista de la SEECI, $\mathrm{n}^{\mathrm{o}}$ 26. Disponible en: www.ucm.es/info/seeci/. [6-6-12]

WRIGHT, C. Christina (1996). Learning disabilities in Mathematics. Disponible en http://www.ldonline.org/article/5947 . [20-9-2010]

\section{Notas}

1 EMAC: "Educació Matemàtica crítica" es un grupo formado por F. Blázquez, A. Darnaculleta, S. Esteve, R. Figueres, J.C. Franquet, A. Miguel, N. Planas y T. Oriol. Son un grupo de maestros y maestras adscritos a la "Associació de Mestres Rosa Sensat" que se han dedicado a la planificación de actividades matemáticas que pretenden estimular el pensamiento crítico 


\section{Los autores}

M. Carme Balaguer Fàbregas es profesora adjunta de la Facultat d'Educació de la Universitat Internacional de Catalunya. Diplomada en Magisterio en la Escuela Universitaria Blanquerna (Universidad Ramon LLull). Licenciada en psicopedagogía (Universitat Oberta de Catalunya). Doctora en Humanidades por la UIC (2009). Premio extraordinario de doctorado (2009). Desarrolla 2 líneas de investigación: influencia de la lectura en el aprendizaje, e implementación del Practicum en los grados de Educación. Coordinadora del ámbito de psicología de la Facultad de Educación de la UIC. Ponente en la Jornadas sobre lectura comprensiva en EDU 21 (Centre d'estudis Jordi Pujol). Tiene diversas publicaciones en distintas revistas de educación.

Salvador Vidal Raméntol es profesor agregado de la Facultad de Educación de la Universidad Internacional de Catalunya, Doctor en Ciencias de la Educación Universidad de Barcelona, UB, (2001). Miembro del Claustro de Doctores de la UB. Miembro del grupo de Investigación SiRSU sobre Sostenibilidad y responsabilidad Social Universitaria de la UIC. Profesor del Máster Universitario en Investigación . Miembro de la Escuela de Doctorado, comisión de Calidad. Vicedecano de la Facultad de Educación (2003-2010). Ha dado capacitaciones sobre competencias matemáticas y didáctica, en distintas Universidades y asociaciones de Iberoamérica, PRORURAL, en Cuzco i Lima en Perú. Con FUNDAP en Quetzaltenango i Guatemala City en Guatemala. Para el ministerio de Educación de Panamá, MEDUCA, para profesores de la Universidad de Panamá. En Argentina, en la Universidad Austral. Miembro de honor del CSEDOHC Consejo Superior Europeo e Iberoamericano de Doctores y doctores Honoris Causa. Tiene distintas publicaciones y destacar el último libro del 2013 El dia del número, motivación de la matemàtica. Saarbrücken: Publicia. 